\title{
Multifunctional Operation of a Fiber Bragg Grating in a WDM/SCM Radio Over Fiber Distribution System
}

\author{
A. Kaszubowska, P. Anandarajah, and L. P. Barry
}

\begin{abstract}
A radio over fiber distribution system incorporating both sub-carrier multiplexing (SCM) and wavelength division multiplexing (WDM) technologies is presented. The SCM signal contains five 155-Mbit/s data channels, centered around $18.5 \mathrm{GHz}$ with $450 \mathrm{MHz}$ spacing. This signal is directly modulated onto three highspeed lasers with emission frequencies spaced by $50 \mathrm{GHz}$. Bragg filters are employed at the receiver base-station in order to both demultiplex the required optical channel and ensure that the detected signal is single sideband (in order to overcome dispersion limitations on the link). Our results show negligible degradation in system performance for the demultiplexing of the WDM signal compared with the back-to-back performance curves.
\end{abstract}

Index Terms-Microwave photonics, optical communications, optical systems, semiconductor laser diode, sub-carrier multiplexing (SCM), wavelength division multiplexing (WDM).

\section{INTRODUCTION}

H YBRID radio/fiber systems are a very attractive option to realize mobile broad-band networks. In these systems, microwave data signals are modulated onto an optical carrier at a central location, and then distributed to remote base-stations using optical fiber. The base-stations then transmit the RF signals over small areas using microwave antennas [1]-[3]. Within these radio networks, it is expected that sub-carrier multiplexing (SCM) will be used for simplifying the complexity of the radio links and the management of the available spectral bandwidth [4]. In addition to SCM, it is also expected that hybrid radio/fiber distribution networks may employ wavelength division multiplexing (WDM) to allow different base-stations to be fed with a common fiber [4]-[6]. In such a system, an optical filter at the base-station will be required to select one of the wavelength channels carrying a specific SCM data signal. The main difficulties with the development of such systems employing SCM and WDM technologies are the cost and complexity of the data generation at the receiver, and the demultiplexing at the base-station. One of the main problems encountered is the need for an optical single-sideband (SSB) signal at the base-station to overcome dispersion effects in the transmission fiber [7]. Previous research in this area has used either complex signal generation techniques to produce SSB signals [4], [5] or demultiplexing techniques using a Fabry-Perot etalon and an array waveguide grating [6], which may be overcomplicated depending on the network architecture that is chosen [4]. For a simple ring

Manuscript received July 15, 2003; revised October 6, 2003. This work was supported in part by the Science Foundation Ireland.

The authors are with the Research Institute for Networks and Communications Engineering, School of Electronic Engineering, Dublin City University, Dublin 9, Ireland (e-mail: liam.barry@dcu.ie).

Digital Object Identifier 10.1109/LPT.2003.821251

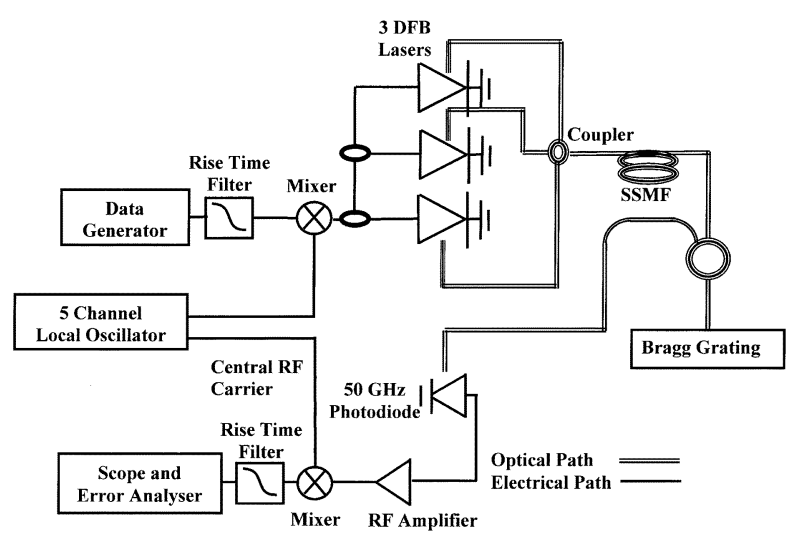

Fig. 1. Experimental setup for hybrid radio/fiber distribution system employing SCM and WDM technologies.

topology, a basic optical filter at the base-station may be the most efficient solution. In this letter, we use direct modulation of a laser diode with the SCM data to generate the optical microwave signal. We also use the WDM technique to achieve a 50-GHz separated three-channel optical double-sideband (DSB) signal. Although the optical signals are DSB, we demonstrate that, by the correct use of a simple Bragg filter at the receiver base-station, we not only can select one of the wavelength channels, but also eliminate one of the sidebands in order to overcome dispersion effects in the transmission fiber [8].

\section{EXPERIMENTAL SETUP}

The experimental setup used is presented in Fig. 1. A 155-Mbit/s nonreturn to zero (NRZ) data stream from an Anritsu pattern generator is initially passed through a $117-\mathrm{MHz}$ low-pass filter to minimize the bandwidth of the data signal. The resulting signal is then mixed with five RF carriers centered at $18.5 \mathrm{GHz}$ (with a channel spacing of $450 \mathrm{MHz}$ ) to generate five binary phase-shift keying (BPSK) data signals. The SCM signal is subsequently split into three ways and used to directly modulate three high-speed (bandwidth $>18 \mathrm{GHz}$ ) single-mode lasers from NTT Electronics. The emission wavelengths of the lasers (around $1550 \mathrm{~nm}$ ) can be slightly altered and set to specific values by temperature control of the diodes. The operating wavelengths chosen are 1549.9, 1550.3, and $1550.7 \mathrm{~nm}$. Even though we use only one data source, the five RF signals are uncorrelated, as we use five separate signal generators that have no phase correlation between them (we are using BPSK modulation, which results in rapid change in the phase of the carrier when the bit value changes). Furthermore, each modulating RF signal is transmitted through different lengths of cable before being applied to the lasers in order to ensure that the three optical channels are uncorrelated. The 

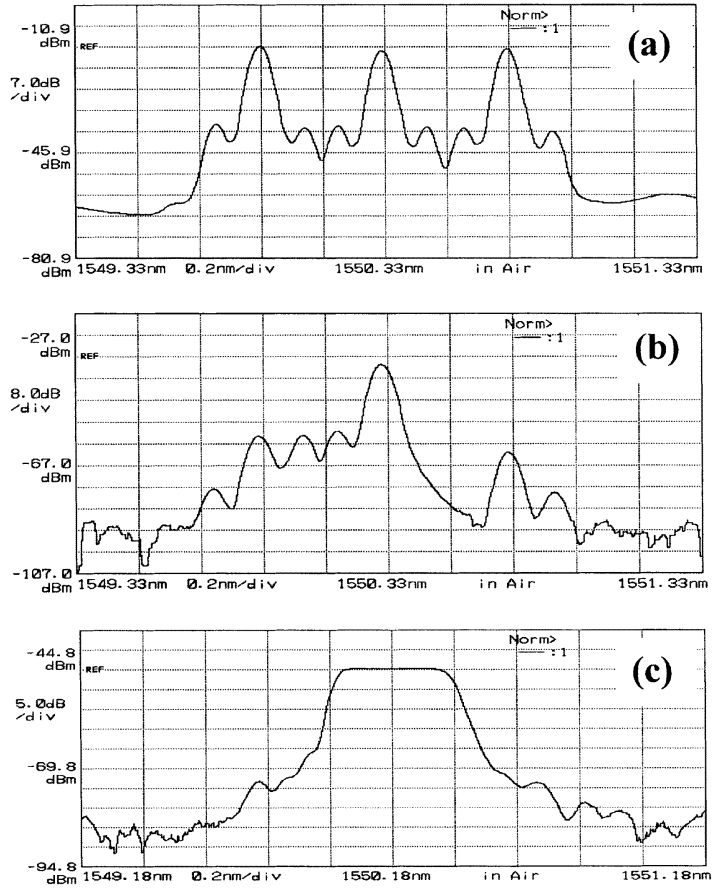

Fig. 2. (a) Optical spectrum of three-channel signal after optical coupler. (b) Reflected spectrum from Bragg filter showing the SSB reflected signal plus adjacent channels. (c) Reflection profile of Bragg grating.

cable lengths for the three signals are $0.25,0.5$, and $0.75 \mathrm{~m}$, respectively, and this corresponds to path differences of over $1 \mathrm{~ns}$ in the cable, which is large enough to uncorrelate the three $18-\mathrm{GHz}$ modulating signals.

With each of the three lasers biased around $60 \mathrm{~mA}$, the SCM signal is applied directly to each of the transmitters without electrical amplification. The optical outputs are then combined using an optical coupler, and selection of one of the optical data channels is carried out using a Bragg filter in conjunction with an optical circulator. The Bragg filters used are designed specifically for WDM systems with 50-GHz spacing, and have reflection bandwidths of approximately $0.35 \mathrm{~nm}$. After selection of one the optical wavelength channels $(1550.3 \mathrm{~nm})$, the signal is then detected using a $50-\mathrm{GHz}$ p-i-n diode. In a complete radio/fiber system, the output signal of the detector would be transmitted through an RF antenna to the mobile network stations where the data is received by down-converting the incoming signal using a local oscillator. However, in our experiment, we have concentrated on the optical part of the system, hence, the down conversion takes place after the photodiode by mixing the SCM data signal with an 18.5-GHz local oscillator. The down-converted signal (central channel of the five-channel $\mathrm{RF}$ signal) is then passed through a low-pass filter to ensure that only the required baseband signal is examined using a $50-\mathrm{GHz}$ oscilloscope and an error analyzer.

\section{EXPERIMENTAL RESULTS}

Fig. 2(a) displays the three-channel optical data signal after passing through the optical coupler. In order to select only one carrier and one sideband of the central optical channel, the Bragg filter is positioned such that the central carrier is at the longest wavelength that is correctly reflected by the Bragg filter. The Bragg filter having a very sharp cutoff ensures that

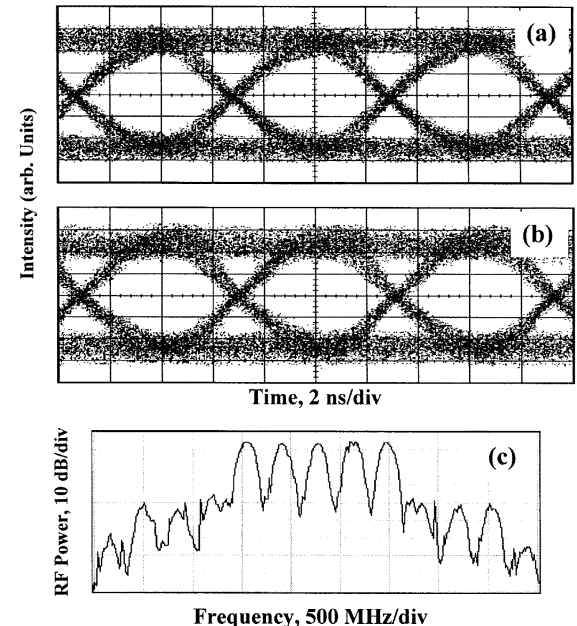

Fig. 3. Received eye diagrams of $155-\mathrm{Mbit} / \mathrm{s}$ data signal with: (a) demultiplexing of WDM signal and (b) back-to-back setup for central optical channel. (c) Electrical spectrum of detected SCM signal.

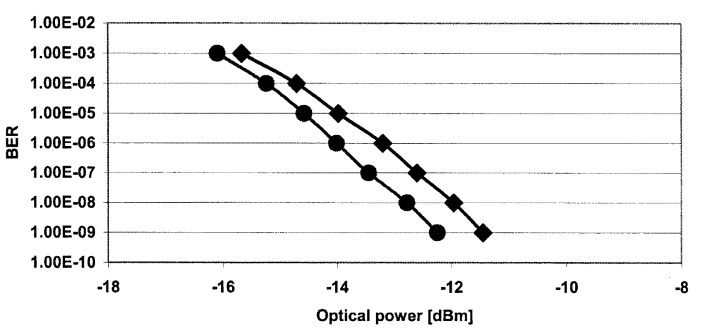

Fig. 4. BER versus received optical power for central RF channel of central optical channel with equal (squares) and unequal (circles) RF channel spacing.

only one sideband is reflected. The reflected signal is illustrated in Fig. 2(b), and we can clearly see that only one sideband of the central channel is reflected by the filter. In addition, the filter also selects out one of the sidebands from the lower wavelength optical channel, and suppresses the carrier of this channel by $27 \mathrm{~dB}$ relative to the power in the central channel. The longer wavelength optical channel is suppressed by $33 \mathrm{~dB}$ relative to the central channel. Fig. 2(c) shows the reflection profile of the filter used for demultiplexing. In terms of its temperature stability, the drift is specified at $0.002 \mathrm{~nm} /{ }^{\circ} \mathrm{C}$, which is small enough to ensure correct operation of the filter in this setup even with temperature fluctuations of up to $10^{\circ} \mathrm{C}$.

Fig. 3(a) displays the received eye diagram after filtering out the central wavelength channel as described above, converting the optical signal to electrical and then down-converting the 18.5-GHz carrier data signal. The intermediate stage, where the optical signal is converted to an electrical signal, is depicted by Fig. 3(c). The received power in this case is $-10 \mathrm{dBm}$. To completely characterize the system performance, two stages of bit error rate (BER) versus received optical power measurements [for the down-converted 155-Mbit/s data signal at an RF carrier frequency of $18.5 \mathrm{GHz}$ (central WDM channel)] were carried out. Firstly, in order to verify the influence of third-order intermodulation distortion (IMD3) on signal performance, we conducted BER measurements for two cases: equal RF channel spacing (IMD3 products interfering with the central RF channel) and uneven channel spacing (such that IMD3 products will not interfere with the central RF channel), as shown in Fig. 4. The equal RF channel spacing used for the first 


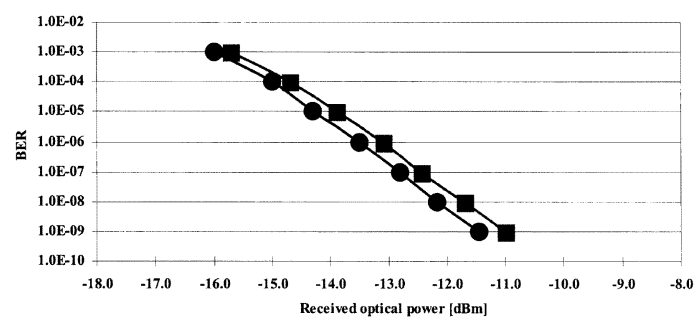

Fig. 5. BER versus received optical power for central RF channel of central optical channel demultiplexed from WDM system (squares) and central optical channel back-to-back (circles).

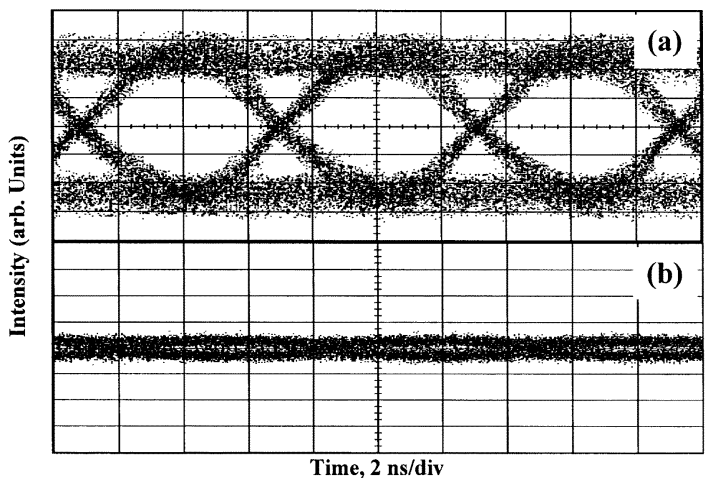

Fig. 6. Received eye diagrams of $155-\mathrm{Mbit} / \mathrm{s}$ data signal after propagation of WDM/SCM signal through $12 \mathrm{~km}$ of standard fiber with the Bragg filter positioned to: (a) select SSB optical signal and (b) select DSB optical signal.

case was $400 \mathrm{MHz}$, while for the unequal channel spacing, we used $550 \mathrm{MHz}$ between the first and second and fourth and fifth channels, and $400 \mathrm{MHz}$ between the second and third and third and fourth channels. We can see that IMD3 causes less than $0.7-\mathrm{dB}$ degradation in system performance. This penalty is due to the dynamic nonlinearity of the laser around the operating $\mathrm{RF}$ band (this has been confirmed by conducting the same experiment around $5 \mathrm{GHz}$, where the dynamic nonlinearity of the laser is negligible, and measuring a penalty of less than $0.1 \mathrm{~dB}$ due to IMD3). Secondly, Fig. 5 displays the system performance when the three optical channels are multiplexed together, and also the back-to-back performance for the central wavelength channel (18.5-GHz electrical channel). We can see from this plot that the degradation in system performance is only of the order of $0.4 \mathrm{~dB}$ due to interference from the adjacent optical channels. The negligible effect of the channel demultiplexing is also clear from Fig. 3(a) and (b), which shows the received eye for the WDM system and the back-to-back case when the received optical power is $-10 \mathrm{dBm}$.

To confirm that our demultiplexing technique does indeed overcome dispersion effects in transmission fiber, we then inserted a 12-km length of standard single-mode fiber after the optical coupler that combines the three wavelength signals. This fiber length has been determined to be that which minimizes the received power of the DSB optical signal due to fiber dispersion effects. Fig. 6(a) displays the received eye diagram for this case. The received optical power level is $-10 \mathrm{dBm}$ and, by comparison with the eye diagrams in Fig. 3, we can see that the degradation in system performance due to the fiber is negligible. We then proceeded to position the Bragg filter such that the carrier of the central wavelength channel is at the center of the filter's reflection band. In this case, the filter selects out the complete DSB optical signal, and the received eye confirms how the fiber dispersion greatly affects the system performance [see Fig. 6(b)].

\section{CONCLUSION}

In this letter, we have demonstrated a hybrid WDM/SCMbased radio-over-fiber distribution system employing direct modulation of the laser transmitters followed by a Bragg filter at the receiver base-station. The system has been used for generation and transmission of a five-channel SCM data signal (each RF carrier modulated with 155-Mbit/s data signal) on three optical wavelength channels spaced by $50 \mathrm{GHz}$. By correct positioning of the Bragg filter relative to the optical channel to be demultiplexed, we can select only the carrier and one sideband in order to overcome dispersion effects in the distribution fiber. Our results show negligible degradation in system performance due to interference between the optical data channels after demultiplexing. This study shows that it may not be a necessity to generate an SSB at the transmitter in a radio/fiber system incorporating WDM technology, as correct optical filtering at the receiver base-station may be used to overcome system limitations imposed by DSB generation at the transmitter. In addition, it should be noted that, to improve the optical spectral efficiency of this technique, it is possible to interleave the optical DSB signals together, two at a time. Depending on the RF carrier frequency used, and the bandwidth of the RF data signal, higher optical spectral efficiencies may be attained by more efficient interleaving of the signals, but this will require more complicated optical demultiplexing technologies at the receiver base-station [9].

\section{REFERENCES}

[1] R. P. Braun, G. Grosskopf, H. Heidrich, C. von Helmolt, R. Kaiser, K Kruger, U. Kruger, D. Rohde, F. Schmidt, R. Stenzel, and D. Trommer, "Optical microwave generation and transmission experiments in the 12- and $60-\mathrm{GHz}$ region for wireless communications," IEEE Trans. Microwave Theory Tech., vol. 46, pp. 320-330, Apr. 1998.

[2] Z. Ahmed, D. Novak, R. B. Waterhouse, and H.-F. Liu, "37-GHz fiberwireless system for distribution of broad-band signals," IEEE Trans. Microwave Theory Tech., vol. 45, pp. 1431-1435, Aug. 1997.

[3] T. Kuri, K. Kitayama, A. Stohr, and Y. Ogawa, "Fiber-optic millimeter-wave downlink system using $60 \mathrm{GHz}$-band external modulation," J. Lightwave Technol., vol. 17, pp. 799-806, May 1999.

[4] G. H. Smith, D. Novak, and C. Lim, "A millimeter-wave full-duplex fiber-radio star-tree architecture incorporating WDM and SCM," IEEE Photon. Technol. Lett., vol. 10, pp. 1650-1652, Nov. 1998.

[5] E. Vourch, D. Le Berre, and D. Herve, "A WDM fiber-radio experiment incorporating a wavelength tunable single-side-band filter," in IEEE MTT-S Int. Microwave Symp. Dig., vol. 3, Seattle, WA, June 2-7, 2002, pp. 1703-1706.

[6] H. Toda, T. Yamashita, K. Kitayama, and T. Kuri, "DWDM demultiplexing with $25 \mathrm{GHz}$ channel spacing for $60 \mathrm{GHz}$ band radio-on-fiber systems," presented at the Eur. Optical Communications Conf., vol. 3, Copenhagen, Denmark, Sept. 8-12, 2002, Paper 8.2.4.

[7] G. H. Smith, D. Novak, and Z. Ahmed, "Overcoming chromatic-dispersion effects in fiber-wireless systems incorporating external modulators," IEEE Trans. Microwave Theory Tech., vol. 45, pp. 1410-1415, Aug. 1997.

[8] C. Marra, A. Nirmalathas, D. Novak, C. Lim, L. Reekie, J. A. Besley, and N. J. Baker, "Optical SSB modulation using fiber Bragg gratings and the impact of grating dispersion on transmission performance," in Int. Microwave Photonics Topical Meeting, Long Beach, CA, 2001, pp. 93-96.

[9] H. Toda, T. Yamashita, T. Kuri, and K. I. Kitayama, "Demultiplexing using an arrayed-waveguide grating for frequency-interleaved DWDM millimeter-wave radio-on-fiber systems," J. Lightwave Technol., vol. 21, pp. 1735-1741, Aug. 2003. 